\title{
Review of: "Directionality of PYD filament growth determined by the transition of NLRP3 nucleation seeds to ASC elongation"
}

li-li $x u^{1}$

1 China Pharmaceutical University Nanjing

Potential competing interests: The author(s) declared that no potential competing interests exist.

In this article, the author describes the cryo-EM structure of a human NLRP3 PYD filament with a resolution of $3.6 \AA$. The authors discovered a unique pattern of highly polar interface residues, which forms isomer interactions, resulting in characteristic filament ends, called A-ends and B-ends, respectively. They combined the drop polymerization test with cryo-electron microscopy and proved that the extension of the ASC adaptor protein on the NLRP3 PYD filament seed is unidirectional and only related to the B-end of the NLRP3 filaments. It is worth noting that NLRP3 and ASC PYD filaments have the same symmetry in the rotation and axial rise of each subunit, allowing continuous transition between NLRP3 as a nucleation seed and ASC as an elongator. This research is very meaningful and highly innovative. This study clearly described the formation of the NLRP3 inflammasome complex in a more concrete and vivid way. It can provide a structural basis for the development of NLRP3 inflammasome formation inhibitors including small molecules, peptides and antibodies. I think this work can be published directly to provide other researchers with an updated research foundation. 